Jaenicke: Untersuchungen über die Secretion d. glandula parotis. 183

\title{
Untersuchungen über die Secretion der glandula parotis.
}

\author{
Von
}

\section{Adolph Jaenicke}

in Königsberg.

Im Sommer 1876 ging ich, angeregt durch Herrn Professor v. Wittich, an die Arbeit, Untersuchungen über die Secretion der Parotis anzustellen und dabei besonders die Stellung des Sympathicus zur Drïse zu berücksichtigen. Die Reihe der Beobachtungen, die bis zu dieser Zeit von den Experimentatoren hierüber angestellt waren, hatte, um sie kurz anzudeuten, folgende Resultate geliefert:

Die Secretion steht ohne Nerveneinfluss still, sie wird angeregt durch Reizung des sympathicus, n. facialis, n. trigeminus und n. glossopharyngeus. Es lässt sich nicht mit Sicherheit entscheiden, ob alle der drei zuletzt genannten Nerven schon von ihrem centralen Ursprunge her sekretorische Fasern der Drìse zuführen, oder ob sie diese erst in ihrem weiteren Verlaufe beigemischt erhalten. Speciell scheint die Absonderung durch den ramus tympanicus glossopharyngei und den $n$. petrosus superficialis minor beherrseht zu werden. Die Einwirkung des sympathicus auf die Drïse lässt sich nicht durch seine vasomotorische Funktion erklären, da die Secretion erst in den weitesten Grenzen von der Blutcirculation in der Drüse abhängig ist. Ferner kann man noch durch Reizung des Grossgehirns und die sensibler Nerven z. B. des centralen ischiadicus-Stumpfes die Secretion einleiten. Das Schaf nimmt in Bezug auf seine parotis eine ganz besondere Stellung ein, da bei ihm die Secretion eine kontinuirliche ist und von keinem Hirnnerven beeinflusst wird. Selbst die. Einwirkung des sympathicus auf die Drüse ist bei diesem Thiere nur sehr zweifelhaft, da eine Reizung des Nerven das Secret nur vorübergehend vermehrt. 
Was nun die Literatur uber diesen Gegenstand betrifft, so hat Nawrocki in seiner Abhandlung „Die Innervation der parotis", Heidenhain's Studien, Heft 4. 1868 eine ausführliche Angabe der betreffenden Arbeiten bis zu seiner Zeit geliefert. Mit Nawrocki's Studien war die Erforschung des Verhältnisses der Nerven zur Secretion der parotis noch lange nicht abgeschlossen, und ich will daher, bevor ich zu meinen eigenen Untersuchungen übergehe, erst noch kurz die nach ihm veröffentlichten Arbeiten anführen:

Vierheller. Beiträge zur Structur und Physiologie der glandula parotis, Zeitschrift für rationelle Medicin von Henle und Pfeufer. Band 31. 1868. Beim Schafe ist die parotis gefässreicher als die glandula submaxillaris. An einem abgebundenen Theile des ductus Stenonianus ist auf Reizung durch den inducirten Strom keine Kontraction zu bemerken. Diese Beobachtung ist tibereinstimmend mit einer Angabe Kölliker's, der bei einer mikroscopischen Untersuchung des Ganges keine glatten Muskelfasern entdecken konnte. Vierheller konstatirt ferner auch eine kontinuirliche Secretion, die nach Durchschneidung des symp. an Grösse nicht abnimmt. Aus einer brieflichen Mittheilung von Schiff an Eckhard geht hervor, dass auch bei der Ziege eine stetige Secretion vorhanden ist, die sich durch Reizung der Mundhöhle nicht deutlich vermehren lässt. Auf Reizung des symp. beim Schafe beobachtet Vierheller eine anfängliche Beschleunigung, die ungefähr $1 / 2$ Minute anhält, dann jedoch schwächer, wie ohne Reizung wird. Bei Reizungen mit Unterbrechungen ist die Summe des in einer bestimmten Zeit secernirten Speichels nicht erheblich grösser, in manchen Fällen sogar kleiner als die Menge des ohne Reizung in derselben Zeit erhaltenen Secretes. Ferner beobachtet Vierheller, dass bei öfterer Reizung des symp. in den Ruhepausen die stetige Secretion ausbleibt. Daraus schliesst er, dass bei Reizung des Nerven das Secret nur durch Verengerung der Bluteapillarmaschen ausgepresst wird.

S. Schröder. Versuche über die Innervation der glandula parotis. Dissertation. Dorpat 1868. Bei Pferden und Hunden giebt die Reizung des vagus-sympathicus einen spärlichen Speichelausfluss aus der parotis, selbst unter curare-Wirkung. Schröder bält auch beim Schafe den symp. für einen Secretionsnerven und 
erklärt die rasche Abnahme des Speichelausflusses bei Reizung des Nerven durch Erschöpfung desselben.

v. Wittieh. Sympathicus und parotis. Berliner klinische Wochenschrift No. 6. 1868.

1) Elektrische Reizung des symp. ruft bei Kaninchen stets ein anfangs schnelles später langsames Ansteigen des Speichels in der Canüle hervor. Eine weitere Steigerung folgt, wenn man nach einer Ruhepause den Nerv von Neuem tetanisirt.

2) Die elektrische Reizung des Hals-sympathicus bleibt vollkommen wirkungslos, wenn der n. facialis derselben Seite ans der Schädelhöhle gerissen wurde, und zwar war der Erfolg ganz der gleiche, wenn der Versuch unmittelbar nach der Ausreissung oder mehrere Tage nach derselben angestellt wurde.

3) Injection des Calabarentractes bewirkt eine profuse Speichelsekretion.

4) Einträufeln eines Tropfens Nicotin in die Lidspalte ruft bei Beginn der Intoxication eine excessive Ausscheidung des parotis-Secretes hervor, die jedoch nicht lange anhaltend bald aufhört. Reizung des symp. bewirkt jetzt weder eine Erweiterung der kontrahirten Pupille, noch Verengerung der Ohrgefässe, noch endlich Secretion der parotis, während die Reizung der Drüse selbst stets von Erfolg begleitet ist.

5) Elektrische Reizung der Drüsensubstanz selbst regt in hohem Grade die Secretion an, wobei es natürlich denkbar bleibt, dass es sich auch hierbei um eine Erregung der peripheren Nervenenden handelt.

6) Centrale Erregung des trigeminus ausgeführt nach Ausreissung des facialis durch intrakranielle Reizung regt energisch die Thätigkeit der parotis an und ist wirksam so lange der Reiz danert.

Grünhagen. Iris und Speicheldrüse. Zeitschrift für rationelle Medicin. Henle und Pfeuffer. Band 33. Dritte Reihe 1868. Die Vermehrung der Parotissecretion bei Calabar-Vergiftung wird durch centrale nicht durch periphere Reizung des trigeminus bedingt. Nicotin auf die conjunctiva bulbi geträufelt bringt Secretion in der parotis hervor nicht reflectorisch durch Reizung der sensiblen Nervenenden in der conjunotiva, sondern ebenso wie calabar durch centrale trigeminus-Reizung. Der symp. hat auf die parotis keinen 
secretionsbefördernden Einfluss, sondern regt nur scheinbar durch Auspressen der acini und Ausführungsgänge die Salivation an.

Eckhard. Der sympathicus in seiner Stellung zur Secretion in der parotis des Sebafes. Eckhard's Beiträge. Band 4. 1869. Eckhard bleibt v. Wittich gegeniber auf seiner alten Anschaung über die Stellung des symp. zur parotis stehn. Es hält es für sehr zweifelhaft, dass dieser Nerv den Process der Absonderung in der Drüse beherrsche.

C. Brettel. Die Parotidensecretion des Schafes im Vergleiche zur Nierensecretion. Eckhard's Beiträge. Band 4. 1869. Brettel beobachtet, dass die Secretion der parotis des Schafes innerhalb sehr weiter Grenzen unabhängig ist von der Geschwindigkeit and dem Drucke des Blutstromes. Beim Schafe dauert die Absonderung noch über eine Viertelstunde nach dem Tode, wenn auch allmälig abnehmend, fort.

L. Loeb. Ueber die Secretionsnerven der parotis und über Salivation nach Verletzung des Bodens des vierten Ventrikels. Eckhard's Beiträge. Band 5. 1870. Loeb kommt durch Versuche bei Hunden zu der Ansicht, dass der ramus tympanicus glossopharyngei der alleinige Sekretionsnerv der parotis sei; ferner beobachtet er bei Verletzung des Bodens des vierten Ventrikels Secretion in den beiden submaxillares, sowie in der parotis der verletzten Seite.

Schwahn. Die Stellung der parotis-Secretion des Schafes zu den Hirnnerven. Eckhard's Beiträge. Band 7. 1876. Beim Schafe wird weder durch Kaubewegungen noch durch Reizung der Mundschleimhant die Menge des parotis-Speichels verändert. Auch bei Durchschneidung des n. facialis dicht nach seinem Austritt ans dem Warzenloch, des n. sympathicus und glossopharyngeus dicht unter dem foramen jugulare, des ramus tympanicus glossopharyngei, des ramus pharyngeus n. vagi, des ramus auriculo-temporalis n. trigemini erfährt sie keine Veränderung. Reizung des nervus facialis verlangsamt die Ausstossung des parotiden-Secretes wahrscheinlich, weil die dadurch hervorgerufene Contraction der Gesichtsmuskeln die Ausfiuhrungsgänge comprimirt. Die parotiden-Secretion ist continuirlich eine nahezu gleich grosse.

Eckhard. Kleinere physiologische Mittheilungen. Eckhard's Beiträge. Band 7. 1876. Eckhard bekommt an nicht eurarisirten Thieren bei Reizung des sogenannten facialis-Kernes im Gross- 
gehirn keine Secretion weder in der parotis noch in der submaxillaris. Erst bei allgemeinem Tetanus tropfte aus allen Drïsen Speichel.

Lépine dagegen „L'influence de l'excitation du cerveau sur la secretion salivaire". Gazette médicale. No. 27. 1875. hatte an curarisirten Hunden bei Reizung der Grossgehirnrinde Secretion in der submaxillaris beobachtet.

Allgemeine Versuchsbedingungen.

Meine Versuche habe ich an Kaninchen, Handen und Schafen angestellt and dabei in keinem Falle curare oder ein narcoticum in Anwendung gebracht. Hat man sich bei den Secretionsversuchen mit dem symp. zu beschäftigen, so ist vor allen Dingen die Curarisirung der Thiere auszuschliessen, nicht nur desshalb, weil das Gift, wie es hin und wieder beobachtet worden ist, die Speichelsecretion vermehrt, sodann besonders wegen seiner Einwirkung auf die vasomotorische Funktion des symp. Wenn auch nach Curareintoxication keine Lähmung dieses Nerven eintritt, so ist doch schon eine schwache Dosis im Stande, eine wesentliche Veränderung des Gefässtonus herbeizuführen. Die betreffenden Angaben hierïber finden wir in Eckhard's Abhandlung "Ueber die Centren der Gefässnerven". Seine Beiträge. Band 7. 1874. pag. $84,87,88$. Von welcher Wichtigkeit es ist, die vasomotorische Funktion des symp. unverändert zu erhalten, werden wir aus der Art seiner Wirkung auf die Secretion kennen lernen. Ebenso misslich ist die Anwendung des Chloroform's und des Morphitum's. (v. Wittich. Virchow's Archiv. Band 37. pag. 97.) Wenn es auch unangenehm ist, an nicht narkotisirten Thieren, besonders Hunden zu operiren, so wird man doch durch die grössere Sicherheit der Beobachtung dafür reichlich entschädigt, zumal, wenn man die Absonderung der Drüse ohne jede künstliche Reizung unter normalen Verhältnissen beurtheilen will. Die Menge des secernirten Speichels bestimmte ich nach Tropfen und zwar zählte ich bei den Reizungsversuchen die Zahl der Sekunden, die die Electroden des Dubois'schen Schlittenapparats den Nerven angelegt werden mussten bis ein Tropfen Speichel von der abwärts gebogenen Caniule abfiel. Es versteht sich von selbst, dass, um die richtigen Werthe bei einer neuen Reizung zu erhalten, erst 
abgewartet wurde, bis nach Wegnahme der Electroden die Nachwirkung des Reizes aufgehört hatte. Dann wurde die letzte Spur von ausgetretenem Speichel von der Mündung der Canüle abgewischt, jetzt erst von Neuem gereizt und die Zeit bis zum $A b$ fallen des nächsten Tropfens bestimmt. Beobachtete ich die Absonderung unter normalen Verbältnissen ohnẹ Reizung, so zählte ich, wenn sie sehr energisch vor sich ging, die Zahl der Tropfen, die in einer bestimmten Zeit abfielen. Zur Einführung in den ductus Stenonianus benutzte ich für Kaninchen öfters Glascaniilen, sonst auch, und bei Hunden und Schafen stets, feine neusilberne Röhren. Um nun auch den Secretionsvorgang beurtheilen zu können, wenn die abgesonderte Speichelmenge so minimal war, dass sie nicht nach Tropfen gezählt werden konnte, schlng ich folgendes Verfahren ein. Es wurde die Metallcanïle an ihrem dicken Ende mit einem ganz kurzen, starkwandigen Kautschukschlauch verbunden und das freie Ende von diesem wieder iiber eine Glasröhre gestreift, die in ein feines und gleichmässiges langes Capillarrohr ausgezogen war, das an seinem äussersten Ende abwärts gebogen wurde. Dieses Röhrensystem band ich mit Wasser vollständig gefüllt in den Speichelgang ein und gab ihm solche Lage, dass keine Knickung des ductus Stenonianus stattfinden konnte. Dann presste ich durch einen gelinden Druck auf den Kautschukschlanch so viel Tropfen zur Glascaniile heraus, dass das Capillarrohr frei von Flüssigkeit beim Nachlassen des Druckes war. Fand eine Absonderung in der Drïse statt, so wurde allmählig die Wassersäule in der feinen Glasröhre nach vorne gedrängt, und ich konnte das Vorschreiten in einer bestimmten Zeit nach Millimetern bestimmen. War das Glascapillarrohr allmälig angefuillt, so konnte ich es durch einen leisen Druck auf den Schlauch wieder entleeren und die Beobachtung von Neuem anstellen. War die Secretion besonders anfangs stark genug, um die Speichelmenge nach Tropfen zählen zu können, so liess ich einfach das Capillarrohr voll laufen und zählte die an der Spitze heraustretenden Tropfen. Die Messungen in dem feinen Glasrohr haben ja natürlich nur einen relativen Werth, und ich wandte sie hauptsächlich bei Beobachtung der normalen Secretion an und wenn es sich darum handelte zu sehen, ob unter bestimmten Bedingungen, besonders bei Kaninchen, Absonderung des Speichels eintritt oder nicht. Für solche Fälle lässt diese Beobachtungsmethode 
an Feinheit nichts zu wünschen übrig. Da das Secret der parotis frei von Mucin ist und wie Wasser tropft (Hoppe-Seyler, Handbuch der physiologisehen und pathologisehen Analyse. 1875. pag. 399), so wird es bei der Zählungsmethode nach Tropfen von keinem wesentlichen Einfluss auî die Grösse desselben beim Abfallen sein, wenn im Laufe des Versuches das Secret sich mit dem wenigen, in der Canüle enthaltenen, Wasser mischt oder schliesslich ziemlich frei von demselben abfliesst.

\section{Die Stellung des sympathicus und vagus zur Secretion der parotis.}

Erste Versuchsreihe.

Ohne anfangs von einer bestimmten leitenden Idee auszugehen, stellte ich zuerst eine Reihe von Versuchen an, die mir die Beobachtungen früherer Experimentatoren auf diesem Gebiete zur Anschauung bringen sollten, in der Hoffnung, dabei vielleicht einen Weg auffinden zu können, auf dem erfolgreich von neuen Gesichtspunkten aus an die Frage über die Stellung des symp. zur Drüse heranzutreten wäre. Dieses geschah, als ich den Einfluss des erhöhten Blutdruckes auf die Secretion prifte. Ich glaubte dabei zu bemerken, dass bei hohem Drucke die Reizung des Nerven nicht so ergiebige Absonderung veranlasste, wie bei der normalen Bluteirculation. Diese Beobachtung war zuerst auf ihre Richtigkeit zu untersuchen und dabei ein Mittel zur Erhöhung des Blutdruckes anzuwenden, das den Organismus nicht unter besonders abnorme Bedingungen brachte. Vagus-Durchschneidung sehien mir nicht rathsam, da dieser Nerv, wie es sich später auch herausstellte, einen Einfluss auf die Secretion ausüben konnte. Ebenso wenig konnte ich durch Unterbindung der einen carotis die Blutcirculation im Gehirn modificiren. Ich schlug folgenden Weg ein. Durch einen möglichst kleinen Schnitt in der linea alba öfnete ich ohne Blutung die Bauchhöhle, isolirte die aorta abdominalis, legte dicht über der Stelle, wo sie sich in die beiden art. iliacae theilt, eine Ligatur lose herum und heftete dann nach Einpackung der Eingeweide die Bauchdecke mit einer Nadel zusammen. 
Versuch vom 21. Oktober 1876.

Kaninchen. Einführung der Canüle in den ductus Stenonianus, Präparation des Hals-sympathicus. Während der Isolirung des Nerven wurde durch die Reizung dabei die vorhernicht vorhandene Secretion eingeleitet. Dann wurde nach Umlegung einer Ligatur der symp. tief am Halse durchschnitten und nun das periphere nach dem Kopfe zu gelegene Ende gereizt, nachdem vorher, wie oben angegeben, die aorta abdominalis zur Unterbindung vorbereitet worden war.

Vor der Unterbindung der aort. abd.

Reizung des symp. in . . . . . . . . . 35 Sec. - 1 Tropfen. Beim Abfall dieses Tropfens wurde die Reizung sistirt

und unter der Nachwirkung derselben fällt der

nächste Tropfen in . . . . . . . . . . 84 Sec. -1 Tropfen. dann der nächste in . . . . . . . . . . . 150 Sec. -1 Tropfen. Darauf wurde die Ligatur um die aort. abd. fest ange-

zogen und es fiel der nächste Tropfen erst in . 9 Min.

Nach der Unterbindung der aort. abd.

Reizung des symp. mit derselben Stromstärke wie oben

gab jetzt in . . . . . . . . . . . . 99 Sec. -1 Tropfen.

Wieder wird bei Abfall des Tropfens die Reizung sistirt

und die Nachwirkung derselben beobachtet. Der

nächste Tropfen fiel in. . . . . . . . 12 Min. 30 Sec.

Darauf kam es überhaupt nicht mehr zur Bildung eines Tropfens.

Versuch vom 25. Oktober 1876.

Kaninchen. Bedingungen wie vorher. Es wurde jetzt aber zuerst die aort. abd. unterbunden.

Nach Unterbindung der aort. abd.

Reizung des symp. in. . . . . . . . . 65 Sec. - 1 Tropfen.

Die Bildung eines zweiten Tropfens wurde unter Nach-

wirkung des Reizes nicht mehr beobachtet. Darauf

wird die Ligatur gelöst.

Ohne Unterbindung der aort. abd.

Reizung des symp. in . . . . . . . . . . . . 20 Sec. -1 Tropfen. Unter Nachwirkung des Reizes fiel der nächste Tropfen in 30 Sec. -1 Tropfen.

Natürlich war hier wieder dieselbe Stromstärke wie vorher bei Unterbindung der aort. abd. in Anwendung genommen.

Diese Zahlen sprechen sehr deutlich. Zunächt ist folgendes zu konstatiren:

1) Nach Unterbindung der aorta abdom. ist, um bei Reizung des symp. einen Tropfen Speichel aus der parotis zu erhalten, eine bedeutend längere Reizdauer nöthig, wie ohne Unterbindung.

2) Ohne Unterbindung der aort. abdom. werden unter Nachwirkung des Reizes auf den symp. noch in verhältnissmässig 
kurzer Zeit einige Tropfen Speichel abgesondert, während bei Reizung des symp. nach Unterbindung der aort. abdom. die Nachwirkung des Reizes zur Bildung eines neuen Tropfens Speichel eine viel längere Zeit in Anspruch nimmt.

Wodurch wird diese Erscheinung bedingt? Sehen wir vor der Hand einmal vollständig von der sogenannten secretorischen Funktion des symp. ab und halten wir uns nur an seine vasomotorische Thätigkeit. Reize ich den Hals-symp., so kontrahiren sich zunächst die Arterien und Arteriolen der betreffenden Kopfhälfte und zwar so, dass die letzteren fast blutleer werden. Es entsteht Ischaemie im arteriellen Stromgebiete. Durch die-Kontraktion der Arterien wird der Blutdruck in dem zu ihnen gehörigen Gebiete der Kapillaren und Venen bedeutend durch fast vollständige Aufhebung der vis a tergo herabgesetzt. Es strömt nun aus dem benachbarten anastomosirenden Venengebiete, in dem normaler Blutdruck herrscht, soviel Blut hinzu, bis die Druckdifferenz sich ausgeglichen. Die Folge davon ist, dass die dem ischaemischen Arteriengebiete entsprechenden Kapillaren und Venen mit angestautem venösem Blute gefüllt werden. So sehen wir in der arteriellen Zone Blutmangel, in den Kapillaren und Venen venöse Stauung bei Sympathicus-Reizung entstehen und die grössere oder geringere Venosität des Blutes wird abhängen von der stärkeren oder schwächeren Reizung des Nerven. Nach Nothnagel's Untersuchungen verläuft ein grosser Theil der vasomotorischen Nerven der Gehirngefässe im Hals-symp. und es liegt daher nahe, dass die bei Reizung des symp. entstehende Stagnation des venösen Blutes im Nervenparenchym einen Reiz auf die Centren der secretorischen Nerven ausübe.

Hier bietet nun die Beobachtung v. Wittich's, dass die Reizung des Hals-symp. vollständig wirkungslos bleibt, wenn der n. facialis derselben Seite aus der Schädelhöhle herausgerissen wurde, einen ausgezeichneten Anhaltepunkt. Zunächst wollte ich mich noch einmal selbst von der Richtigkeit dieser Beobachtung: iiberzeugen, ehe ich in meinen Folgerungen weiter ging.

Um sicher sein zu können, dass der $n$. facialis auch vollständig von seinem Ursprunge losgetrennt wurde, schlug ich einen anderen Weg ein. Bei einem Kaninchen entfernte ich das Schädeldach, nachdem ich mich zuvor iiberzengt hatte, dass die Reizung. des freigelegten symp. eine energische Secretion einleitete. Darauf 
trug ich das Grossgehirn soweit ab, dass ich sicher den n. facial. an seinem Ursprunge durchschneiden konnte. Nach der Durchtrennung des Nerven war jedes Mal die Reizung des symp. durch schwache oder starke Ströme ohne Erfolg, während die elektrische Erregung des peripheren facialis-Stumpfes noch immer die Secretion sehr wirksam anregte. Die Blutung wurde bei der Operation durch Anwendung von Schwämmen and Eisenchlorid möglichst gestillt und das Thier so bis zum Ende des Versuches am Leben erhalten.

Darauf stellte ich einen zweiten Versuch nach der v. Wittich'schen Methode an. Nach Einfuihrung der Kanüle in den Speichelgang wurde bei einem Kaninchen der freigelegte symp. elektrisch gereizt und dabei seine Wirksamkeit konstatirt. Darauf riss ich den n. facial. aus der Schädelhöhle aus. Durch die Reizung des Nerven bei der Operation fallen 2 Tropfen Speichel. Nachdem ich mich nach einer Pause davon überzengt hatte, dass die Secretion gänzlich stillstand, reizte ich den symp. anfangs mit schwachen, dann immer stärkeren Strömen, aber in keinem Falle mit auch nur dem geringsten Erfolge. Da ich den Stand der Speichelsäule in dem Glaskapillarrohr beobachtete, musste die Bestimmung ganz genau sein.

Durch beide Versuche konnte ich also die Beobachtung v. Wittich's bestätigen.

Es bleibt demnach die Möglichkeit völlig ausgeschlossen, dass der symp. Fasern führen kann, die direkt secretorisch wirken. Dieser Nerv steht in seiner Wirkung auf die Speichelsecretion vielmehr in dem innigsten Zusammenhange mit dem centralen Ursprunge des n. facial. und es bleibt nur uibrig, die Frage zu erörtern, ob der symp. reflektorisch auf den facial. einwirken kann oder ob bei seiner Reizung nur allein die obenerwähnten Vorgänge gleichzeitig das facialis-centrum in einen Erregungszustand versetzen. Wollten wir hier einen reflectorisch wirkenden Vorgang annehmen, so begegnen wir den grössten Schwierigkeiten, wenn wir das Experiment mit der Unterbindung der aort. abdom. in dieser Beziehung zu deuten suchen wollten. Es lässt sich nicht absehen, wie durch eine grössere Blutfülle in dem secretorischen Apparat eine Verlangsamung des Reflexvorganges verursacht werden kann. Ich erkläre mir die Einleitung der Speichelsecretion bei Reizung des symp. auf die oben angedeutete Weise. Die durch 
Kontraction der Arterien bewirkte Stagnation des venösen Blutes setzt die Centren der secretorischen Nerven in einen Erregungszustand, und es beherrscht somit der symp. nur durch seine vasomotorische Funktion die Absonderung der parotis. Halten wir dieses fest, so erklärt sich das Experiment mit der Unterbindung der aort. abdom. von selbst. Wenn bei Reizung des symp. die Gefässmuskulatur sich kontrahirt, so hat sie eine Arbeit zu leisten, nämlich den normalen Blutdruck zu überwinden. Unterbinde ich die aort. abdom., so erhöhe ich den Blutdruck und kontrahirt sich jetzt die Gefässmuskulatur, so hat sie eine grössere Arbeit wie vorher bei normalem Druck zu vollziehen, wenn sie denselben Effekt erzielen will. Soll die Muskulatur aber mehr leisten, so muss ich den ihr zugehörigen Nerven entweder stärker oder längere Zeit hindurch reizen. Wende ich, wie es bei dem Experiment geschah, zur Reizung dieselbe Stromstärke an, so werde ich nach Unterbindung der aort. abdom. eine längere Reizdauer brauchen, um dieselbe Menge Speichel zu gewinnen, wie vor der Unterbindung. Denn unter Anwendung derselben Stromstärke werden bei erhöhtem Blutdruck die Gefässe sich nicht so energisch kontrahiren, wie bei normalem Druck. Die Stauung des venösen Blutes wird also schwächer ausfallen und auch einen geringeren Reiz für die Secretionsnerven abgeben.

\section{Zweite Versuchsreihe.}

Versuch vom 4. Dezember 1876.

Kaninchen. Einführung der Kanüle in den ductus Stenonianus. Präparation des Hals-symp. Die Reizung begann, als vollständiger Secretionsstillstand eingetreten war.

Reizung des symp. in . . . . . . . . . 30 Sec. -1 Tropfen. Reizung des symp. in. . . . . . . . . 33 Sec. -1 Tropfen.

Durchschneidung des symp.

Reizung des peripheren symp. in. . . . . . . 120 Sec. -1 Tropfen. Reizung des peripheren symp. in. . . . . . 80 Sec. -1 Tropfen. Reizung des peripheren symp. in. . . . . . . . 80 Sec. -1 Tropfen.

Es versteht sich von selbst, dass die Reizung stets in denselben Intervallen und mit derselben Stromstärke unternommen wurde.

Versuch vom 23. März 1877.

Kaninchen. Reizung des freigelegten symp. in. 55 Sec. - 1 Tropfen. Reizung des symp. in . . . . 60 Sec. -1 Tropfen. 
Durchschneidung des symp.

Reizung des peripheren symp. ergab in $2 \% / 2$ Minute einen kleinen Tropfen, der jedoch in dieser Zeit noch nicht eine solche Grösse erhalten hatte, dass er abfallen konnte. Bis hierher war die Reizung ausgeführt bei einem Rollenabstande von $13 \mathrm{~cm}$. Ich erhöhte hierauf die Stromstärke durch Annäherung der Spiralen bis auf $9,5 \mathrm{~cm}$, obne dass ich bei einer Reizdauer von 2 Minuten einen Tropfen von der Grösse erhielt, dass er abfallen konnte. Selbst bei Rollenabstand $8,5 \mathrm{~cm}$ erhielt ich bei einer Reizdauer von $2 \mathrm{Minu}-$ ten 40 Sekunden noch keinen abfallenden Tropfen.

Die Versuche wurden an Thieren ausgefuihrt, bei denen ohne Reizung nicht die geringste Absonderung konstatirt werden konnte. Es kann also keines Falls ein secretoriseher Tonus des symp. angenommen werden, denn wo keine Wirkung vorhanden ist, kann anch kein Tonus bestehen, und dennoch sehen wir, dass nach Durchschneidung des symp. eine anffallend längere Reizdauer nothwendig ist, um denselben Effekt zu erzielen. Man könnte vielleicht sagen, dass die Durchschneidung des Nerven als ein so intensiver Reiz wirkt, dass er die elektrische Erregbarkeit desselben auf längere Zeit herabsetzt. Dieser Annahme steht aber entgegen, dass die Durchschneidung des Nerven ein verhältnissmässig schwacher Reiz ist, der oft nicht den geringsten Einfluss auf die Absonderung ausiubt, in anderen Fällen nur einen sehwachen. Jedenfalls wirkt die Zertrennung nie so anhaltend, wie selbst ein schwacher elektrischer Strom und nach diesen Reizungen können wir innerhalb der nothwendigen Ruhepausen niemals eine so schnelle Abnahme der Erregbarkeit des Nerven beobachten. Nach Durchschneidung des Hals-symp. tritt aber sofort eine bedentende Gefässdilatation in den oberhalb gelegenen Theilen ein, weil durch Lostrennung der tiefer gelegenen Hals- und Brustganglien der Nerv einen Theil seines kontinuirlichen Tonus verliert und diesen Verlust muss man nach der Durchsehneidung bei seiner elektrischen Erregung durch eine längere Reizdauer oder durch eine grössere Stromstärke ersetzen, wenn man den gleichen vasomotorischen Effekt und in Folge dessen auch den secretorischen erzielen will. Hierin findet das Experiment seine Erklärung.

\section{Dritte Versuchsreihe.}

In weiterer Verfolgung meiner Hypothese stellte ich noch folgende Versuche an. Bei Kaninchen reizte ich nach Einführung des Glaskapillarrohrs in den Speichelgang den symp., um mich bei jedem Thiere von seiner Ein- 
wirkung auf die Absonderung zu überzeugen. Dann liess ich das Thier durch Anschneiden der Carotiden verbluten und konnte bei den Verblutungskrämpfen und noch längere Zeit nach ihnen ein allmähliges Ansteigen der Speichelsäule in dem Kapillarrohr beobachten. Nach den Verblutungskrämpfen aber noch zu einer Zeit, wo ein deutliches Ansteigen des Speichels zu beobachten war, war die Reizung des symp. nicht mehr im Stande die Menge des in einer bestimmten Zeit abgesonderten Secretes zu vermehren. Durch Reizung des Hals-symp. bewirkte ich eine Verminderung der Zufuhr von arteriellem Blut in das Gehirnparenchym, durch Verblutung erreiche ich denselben Effekt und so musste ich auch bei der Verblutung eine Secretionssteigerung beobachten. Waren die arteriellen Gefässe erst ihres Inhaltes beraubt worden, so durfte auch die Reizung des Hals-symp. keine Steigerung der Absonderung hervorrufen, wenn meine Annahme, dass der symp. keine secretorischen Fasern. führt, richtig ist. Das Ausbleiben des Erfolges kann man wohl nicht auf ein Erlöschen der Erregbarkeit der secretorischen Fasern schieben, denn in allen Fällen überzeugte ich mich davon, dass die intrakramelle Reizung der secretorischen Nerven noch eine Vermebrung des Secretes herbeizuführen im Stande war.

\section{Vierte Versuchsreihe.}

Es wurde bei Kaninchen nach Einführung der Kanüle in den duct. Stenon. und nach Präparation des Hals-symp. eine Trachealfistel angelegt und in diese zur Einleitung der künstlichen Respiration eine Glasröhre eingebunden.

Versuch vom 26. Oktober 1876.

Kaninchen.

Reizung des symp. in. . . . . . . . . . 20 Sec. -1 Tropfen.

Reizung des symp. in . . . . . . . . . 30 Sec. -1 Tropfen.

Eirleitung starker künstlicher Respiration.

Reizung des symp. in. . . . . . . . . . . 80 Sec. - 1 Tropfen.

Der Schlauch, der den Blasebalg mit der in die Trachea eingebundenen Glasröhre verband, wird von letzterer abgezogen und die künstliche Respiration sistirt.

Reizung des symp. in. . . . . . . . . . 20 Sec. -1 Tropfen.

Bis zur nächsten Reizung mit gleichzeitiger künstlicher Respiration gönnte ich dem Nerven eine Ruhepause von 10 Minuten.

Reizung des symp. mit künstlicher Respiration in. . 115 Sec. - 1 Tropfen. Nur 3 Minuten später Reizung des symp. ohne künst-

liche Respiration in . . . . . . . . . 55 Sec. -1 Tropfen.

Versuch vom 15. November 1876.

Kaninchen.

Reizung des symp. ohne künstliche Respiration . . 45 Sec. -1 Tropfen. Reizung des symp. mit kïnstlicher Respiration in . $\quad \mathbf{3}_{1 / 2}^{1 / 2}$ Min. - 1 Tropf. Reizung des symp. ohne künstliche Respiration in . 40 Sec. - 1 Tropfen. 
Dieser letzte Versuch ergab noch ein anderes interessantes Resultat. Ich wollte das Thier am Leben erbalten, nähte desshalb die Trachealfistel zu, dabei entstand starke Dyspnoë mit gleichzeitiger starker Speichelsecretion. In dem Dezember-Hefte des Pflüger'schen Archiv's 1876 hat inzwischen Luchsinger ${ }^{1}$ ) mitgetheilt, dass er bei Erstickungskrämpfen durch Verschluss der Trachea Secretion der glandula submaxillaris beobachtet habe und erklärt diese Erscheinung dadureh, dass das Erstickungsblut die Centren der Secretionsnerven reizt. Dieselbe Erscheinung beobachtete er anch bei starker Wärme-Dyspnoë. Hier finden wir also eine Analogie zwischen beiden Speicheldrüsen.

Gehen wir nun auf die Versuche mit der kïnstlichen Respiration zuriick, so können wir hier eine ähnliche Erscheinung konstatiren, wie bei den Versuchen mit der Unterbindung der aort. abdom. Es fragt sich nun, $o b$ und wie diese Erscheinung: mit meiner Hypothese von der Einwirkung des symp. auf die Speichelsecretion in Einklang zu bringen ist. Wir haben gesehen, dass das stagnirende venöse Blut die Secretionsnerven reizt. Einen solchen Reiz übt es aber nur durch seinen bedentenden Kohlensäuregehalt aus, und in dem Maasse, in dem dieser zunimmt, d. h. je venöser das Blut wird, wird auch die Absonderung des Speichels vermehrt. Wenn bei Reizung des symp. mangelhafte Blutzufuhr im arteriellen Gebiete und venöse Stagnation in den Kapillaren and Venen stattfindet, so wird dieses bei einem bestimmten Erregungsgrade des Nerven einen bestimmten Reiz auf die secretorischen Centren ausüben. Machen wir durch künstliche Respiration das arterielle Blut ärmer an Kohlensäure, so wird, wenn wir den symp. mit derselben Stromstärke dieselbe Zeit hindurch wie vorher reizen, auch in demselben Grade die Blutzufuhr vermindert werden und derselbe verlangsamte Abfluss des venösen Blutes wie vorher statthaben. 'Da aber das wenige, durch die Arterien zuströmende Blat sehr viel Kohlensäure ärmer ist, wie ohne künstliche Respiration, so wird sich dieses Moment auch noch in den Kapillaren und Venen geltend machen, d. h. das venöse Blut wird bei der künstlichen Respiration mit gleichzeitiger Sympathi-

1) B. Luchsing er, Pflüger's Archiv, 14. Band, 8. u. 9. Heft, pag. 389. December 1876. "Weitere Versuche and Betrachtungen zur Lehre von der Nervencentren". 
cus-Reizung eine geringere Venosität, also einen geringeren Kohlensäuregehalt besitzen, wie vorher bei Symp.-Reizung ohne künstliche Athmung. Reizung des symp. und küinstliche Respiration wirken also entgegengesetzt auf die Centren der secretorischen Nerven.

So fand ich auch in diesem Experiment eine bedeutende Stütze für meine Anschaung über die secretorische Funktion des symp.

Dieser Versuch erinnert mich an eine Beobachtung von Rosenthal und Uspensky. Reizt man den symp., so wirkt dieser reflektorisch auf den vagus ein, und es kommt Verlangsamung des Herzschlages zu Stande. Diese beiden Forscher fanden nun, dass bei Reizung des symp. mit gleichzeitiger künstlicher Respiration die Herzverlangsamung entweder gar nicht auftritt oder doch eine bedeutend schwächere wird, wie bei Reizung des Nerven ohne künstliche Respiration und zogen daraus den Schluss, dass künstliche Respiration die Reflexerregbarkeit speciell im Gebiete des symp. herabsetzt ohne jedoch angeben zu können, wie ein solcher Vorgang zu Stande kommen kann. Für diesen Fall glaube ich, dass mein Experiment mit. der künstlichen Respiration im Gebiete der Speichelsecretion genügende Aufklärung geben kann. Meiner Ansicht nach liegt hier dasselbe Verhältniss zwischen symp. und ragus vor, wie es meine Versuche zwischen symp. und facialis konstatirt haben.

\section{Fünfte Versuchsreihe.}

Als ich soweit mit meinen Beobachtungen gekommen war, hatte meine Hypothese noch eine schwere Priffung zu bestehen. Czermak hatte gefunden, dass die elektrische Reizung des Halssymp. beim Hunde die Speichelabsonderung der submaxillaris unter gewissen Umständen verlangsamen und sogar aufheben kann. Heirdenhain bestätigte diese Beobachtung and nahm an, dass der symp. Secretionsbefördernde, und von diesen sogar noch zwei verschiedene Sorten, und Secretionshemmende Fasern führe. Es war nun vor allen Dingen nothwendig zu untersuchen, ob auch bei der parotis die Reizung des symp. die Secretion unter Umständen verlangsamen kann. Beim Kaninchen konnten diese Beobachtungen natïrlich nicht angestellt werden, da ich einen hemmenden Einfluss des symp. doch nur auf eine Secretion erwarten 
konnte, die nicht durch ihn selbst hervorgebracht war. Während der Verdaung findet anch beim Kaninchen eine continuirliche doch allmählig abnehmende Absonderung von Speichel statt, die jedoch so schwach ist, dass sie sich nicht gut zu einem Versuche eignet. Beim Hunde ist diese Speichelsecretion während der Verdauung bedeutend stärker, sodass man hier an einem guten $\mathrm{Ob}$ jecte die Menge des secernirten Speichels bequem nach Tropfen messen kann.

Versuch vom 1. December 1876.

Hund, der vorher stark gefüttert war. Starke Verdauungs-Speichelsecretion. Nach Einführung der Kanüle in den duct. Stenon. wird die Speichelmenge bestimmt.

$$
\begin{array}{llll}
7 & \text { Tropfen in } 1 & \text { Min. } \\
7 & \text { in } 1 & \text { Min. } \\
5 & \# & \text { in } 1 & \text { Min. }
\end{array}
$$

Jetzt wird der gemeinschaftliche vagus-symp. tief am Halse präparirt. Dabei fallen

$$
\begin{aligned}
& 2 \text { Tropfen in } 1 \mathrm{Min} . \\
& 3 \% \text { in } 1 \text { Min. } \\
& 3 》 \quad \text { in } 1 \text { Min. } 10 \text { See. } \\
& 3 \% \text { in } 1 \text { Min. }
\end{aligned}
$$

Darauf wird der vagus-symp. durchschnittten

2 Tropfen in 1 Min. 30 Sec.

$2 \quad " \quad 1$ Min. 15 Sec.

$3 \quad$ " 1 Min. 15 Sec.

3 " "1 Min.

3 " 1 Min.

Jetzt wird der gemeinschaftliche centrale vagus-sympathicus - Stumpf gereizt. Es fällt dabei

1 Tropfen in 1 Min. 10 Sec.

1 " $1 \mathrm{Min} .20 \mathrm{Sec}$.

Dann wird die Reizung sistirt und es fallen jetzt

4 Tropfen in 1 Min. 10 Sec.

Reizung des centralen vagus-sympathicus-Stumpfes mit etwas stärkerem Strome

1 Tropfen in 1 Min. 30 Sec.

Ohne Reizung:

3 Tropfen in 1 Min. 10 Sec.

Es wird jetzt der periphere vagus-sympathicus-Stumpf aufgesucht und unterbunden. Dann wird die Menge des ohne Reizung secernirten Speichels bestimmt.

1 Tropfen in $1 \mathrm{Min}$.

1 " 11 Min.

$1 " \quad$ "1 Min. 
Reizung des peripheren vagus-symp.

$$
5 \text { Tropfen in } 1 \text { Min. }
$$

Ohne Reizung:

$$
\begin{aligned}
& 4 \text { Tropfen in } 1 \text { Min. } \\
& 4 \quad " \quad 1 \text { Min. } 5 \text { Sec. }
\end{aligned}
$$

Nach einigen Minuten:

$$
2 \text { Tropfeir in } 1 \mathrm{Min} .
$$

Reizung des peripheren vagus-symp.

1 Tropfen in 3 Min.

Ohne Reizung:

1 Tropfen in 1 Min. 25 Sec.

2 " 1 Min. 40 Sec.

Nach 5 Minuten gemessen:

1 Tropfen in $1 \mathrm{Min}$.

Reizung des peripheren vagus-symp.

3 Tropfen in 1 Min. 5 Sec.

Ohne Reizung:

3 Tropfen in 1 Min. 25 Sec.

$3 \quad " \quad 1$ Min. 50 Sec.

2 " $2 \mathrm{Min}$.

Reizung des peripheren vagus-symp.

5 Tropfen in 1 Min. 15 Sec.

Ohne Reizung:

3 Tropfen in . . $55 \mathrm{Sec}$.

Reizung des centralen vagus-symp.:

1 Tropfen in 5 Min.

Ohne Reizung:

2 Tropfen in 1 Min.

$2 \quad$ " 1 Min.

$2 " \quad n \quad 57$ Sec.

$1 " n \quad 35 \mathrm{Sec}$.

$1 " \quad " \quad 28 \mathrm{Sec}$.

$1 n \quad 35 \mathrm{Sec}$

Reizung des centralen vagus-symp. 1 kleiner Tropfen in 90 Sec., der durch Bewegung des Thieres abgeworfen wurde.

Ohne Reizung:

1 Tropfen in 20 Sec.

$1 " \quad$ " 30 Sec.

$1 \quad$ $\quad 1$ Min. 4 Sec.

$1 \quad " 1 \mathrm{Min}$.

$1 " \quad " \quad 31$ Sec.

$1 ", \quad 30 \mathrm{Sec}$.

$\downarrow " \quad 20$ Sec. 
Betrachten wir nun näher die Ergebnisse dieses Versuches. Ueber die Speichelsecretion während der Verdauung und die Art und Weise, wie sie zu Stande kommt, werden wir später sprechen. Augenblicklich interessirt uns nur das Verhältniss des symp. zu ihr. Am Anfange des Versuches fielen durchschnittlich 7 Tropfen in 1 Min. Als ich den vagus-symp. präparirte, trat durch die dabei unumgängliche mechanische Reizung des Nerven bereits eine bedeutende Verlangsamung der Speichelsecretion ein, die nach Beendigung der Operation wieder anstieg. Es war also ersichtlich, dass bereits mechanische Reizung des vagus-symp. eine Verlangsamung der Speichelsecretion herbeiführen kann und so musste analog der Czermak'sehen Beobachtung für die submaxillaris der symp. auch hemmende Fasern für die parotis fubren. Es war nun noch zu entscheiden in wie weit der periphere oder der centrale Theil des vagus-symp. eine Rolle spielen. Desshalb durchsechnitt ich den vagus-symp. und es zeigte sich dabei, dass auch die Durchschneidung des Nerven als ein Reiz wirkt, der die Secretion verlangsamt. Einige Zeit nach der Durchsehneidung nahm die Secretion wieder zu zum Beweise, dass die Zertrennung als Reiz zu wirken aufhörte. Nun ergiebt das Versuchsprotokoll, dass bei Reizung des centralen vagus-sympathicus-Stammes stets eine bedeutende Verlangsamung der Absonderung stattfand, während die Reizung des peripheren Endes theils die Secretion beschleunigte, theils gar keine bedeutende Modifizirung herbeirief. Durch die Angabe früherer Beobachter stand es fest, dass auch bei Hunden die Reizung des vagus-symp. die Speichelsecretion einleitete. Ich selbst hatte dieses auch mehrfach beobachtet. Da keiner von den Experimentatoren mit alleiniger Ausnahme von $0 \mathrm{ehl}$ bei Reizung des vagus Speichelsecretion erhalten hatte, so war es klar, dass allein der symp. die Absonderung anregen konnte. Auch ich habe an Versuchen bei Kaninchen durch Reizung des ragus niemals Speichelsecretion auftreten sehen. Desshalb hatte man sich die doch an und für sich so sehr zweifelhafte Trennung des vagus vom symp. im grossen und ganzen erspart, wenn auch hin und wieder der Versuch einer isolirten Reizung gemacht worden ist. Als ich durch meine Versuche gefunden hatte, dass die Reizung des gemeinschaftlichen vagus-sympathicus-Stammes bei der parotis die Speichelsecretion während der Verdauung zu verlangsamen im Stande ist, schien es mir unzweifelhaft nöthig nach Czermak's 
und Heidenhain's Vorgang für die submaxillaris anch für die parotis im symp. secretionshemmende Fasern annehmen zu müssen. Nun hatte ich aber auf Grund meiner vorhergehenden Versuche die Hypothese aufgestellt, dass der symp. überhaupt keine secretorische Fasern führen kann und dieser Auffassung stand nun der secretionshemmende Einfluss des Nerven schroff gegenüber. War es wirklich der symp., der die Secretion hemmte, so musste ich meine Hypothese aufgeben. Von dem vagus war es nur als feststehend zu betrachten, dass er die Secretion nicht beförderte und wenn beim Hunde bei Reizung des vagus symp. zusammen eine Verlangsamung der Speichelsecretion während der Verdautng eintrat, so lag doch die Vermuthung nahe, dass in dem ragus selbst vielleicht diese Hemmungswirkung zu suchen sei. Aber wie diese Versuche anstellen? Bei dem Hunde den vagus vom symp. sicher isoliren zu wollen schien mir zu unverlässig und ich benutzte deshalb zu meinen Versuchen hierüber das Kaninchen, weil bei ihm beide Nerven voneinander getrennt liegen. Um nun hier einen etwaigen hemmenden Einfluss des vagus auf die Absonderung zu prüfen, benutzte ich nicht die bei diesem Thiere so schwache Secretion während der Verdauung, sondern die Absonderung bei sympathicus-Reizung.

Versuch vom 4. December 1876.

Kaninchen. Nach Einführung der Kanüle in den Speichelgang und nach Isolirung des vagus und symp. wurde die Reizung des letzteren mit Erfolg auf die Speichelsecretion geprüft. Dann durchschnitt ich den vagus und reizte das centrale Ende desselben, ohne dass Absonderung eintrat.

Reizung des unzertrennten symp. und des centralen vagus-Stumpfes zusammen:

1 Tropfen in 40 Sec.

Reizung des symp. allein:

1 Tropfen in 30 Sec.

Reizung des symp. und des centralen vagus-Stumpfes zusammen:

1 Tropfen in 45 Sec.

Reizung des symp. allein:

1 Tropfen in 33 Sec.

Durchschneidung des symp.

Reizung des peripheren nach dem Kopfe zu gelegenen Theiles des symp.:

1 Tropfen in $2 \mathrm{Min}$.

E. Pfüger, Archiv f. Physiologie. Bd. XVII. 
Reizung des centralen vagus and des peripheren symp. zusammen:

1 Tropfen in 4 Min. 15 Sec.

Reizung des peripheren symp. allein:

1 Tropfen in 1 Min. 20 Sec.

Bei gleichzeitiger Reizung der Nerven hatte ich diese zusammen auf die Elektroden gelegt und um nun dem Einwande zu entgehen, dass die gleichzeitige Auflegung des vagus die Reizung des symp. schwächer ausfallen lassen könnte, reizte ich den peripheren symp. mit dem peripheren vagus-Ende zusammen und erhielt

\section{Tropfen in 1 min. 20 sec.,}

also genau denselben Werth, wie vorher bei Reizung des symp. allein. Es konnte also die Verlangsamung der Secretion bei Mitreizung des centralen vagus nicht durch Theilung der Stromstärke entstanden sein, sondern allein durch die physiologische Einwirkung des centralen vagus-Stumpfes auf die Absonderung. Um jedoch noch sicherer zu gehen, beschloss ich die Reizung des vagus und symp. auf zwei verschiedenen Elektrodenparen getrennt von einander zu unternehmen.

Versuch vom 24. März 1877.

Kaninchen. vagus and symp. werden undurchschnitten gereizt, um nicht nach Durchschneidung des symp. grössere Stromstärken in Anwendung bringen zu müssen. Reizung des symp. allein:

1 Tropfen in 45 Sec.

Reizung des vagus und symp. zugleich auf zwei verschiedenen Elektrodenpaaren.

Reizung des vagus wurde bei Rollenabstand $7,5 \mathrm{~cm}$. ausgeführt:

1 Tropfen in 45 Sec.

Die Reizung des vagus schien jedoch zu schwach gewesen zu sein und es wurde desshalb eine grössere Stromstärke angewandt.

Reizung des symp. allein:

$$
1 \text { Tropfen in } 35 \text { Sec. }
$$

Reizung des vagus und symp. auf zwei verschiedenen Elektrodenpaaren. Reizung des vagus wurde bei einem Rollenabstand von $8,5 \mathrm{~cm}$ mit einem anderen stärker wirkenden Schlittenapparat ausgeführt. In 2 Minuten hatte sich erst ein Tropfen gebildet, der jedoch noch nicht abfiel, dann hörte ich mit der Reizung auf und selbst nach weiteren 2 Minuten hatte sich unter der Nachwirkung der Reizung der Tropfen noch nicht soweit vergrössert, dass er abfallen konnte. 
Reizung des symp. allein:

1 Tropfen in 1 Min. 40 Sec.

Die Nachwirkung der Reizung war hier sebr sichtbar.

Reizung des symp. allein:

1 Tropfen in 1 Min. $45 \mathrm{Sec}$.

Reizung des vagus und symp. Stromstärken wie vorher:

1 Tropfen in 3 Min. 15 Sec.

Aus dem Versuche am Hunde ersahen wir, dass bei Reizung des centralen vagus-sympathicus-Stumpfes eine Verlangsamung der Verdauungsspeichelsecretion eintrat. Der Versuch am Kaninchen entscheidet, dass nicht der symp., sondern die centripetalleitenden Fasern des vagus secretorische Hemmungsnerven sind. Ich konnte demnach meine Hypothese von dem Verhältniss des symp. zur Speichelabsonderung aufrecht erhalten und gerade diese Hypothese war es, die mich zur Auffindung dieser so interessanten Funktion des vagus führte. Wenn ich auch dabei stehen bleiben konnte, dass der symp. keine secretorischen Fasern führt, so trat ich beim vagus einem secretorischen Hemmungsmechanismus entgegen. Zunächst war zu sehen, ob eine Erklärung für den hemmenden Einfluss des vagus za finden möglich war. Die Hemmungsmechanismen im thierischen. Organismus sind ja fast die dunkelsten Punkte im ganzen Gebiete der Physiologie. Beim Hunde sahen wir, dass der vagus die Speichelsecretion während der Verdauung hemmt, beim Kaninchen war dieser hemmende Einfluss des Nerven auch bei Reizung des symp. zu finden. Der symp. leitet die Secretion ein, der vagus hemmt sie, also müssen wir beide in Bezug auf die Speichelsecretion als Antagonisten betrachten. Ruft der symp. die Speichelsecretion durch seine vasomotorische Funktion hervor, so liegt es nahe, dass auch sein Antagonist in secretorischer Beziehung die Hemmung durch eine und zwar entgegengesetzte vasomotorische Funktion zu Stande bringt. Dass eine solche Funktion diesem Nerven zukommt, ist eine bekannte Thatsache. Der vagus giebt bei manchen Thieren nicht nur einen besonderen ramus depressor $a b$, sondern er führt anch im Stamme selbst Fasern, die centripetal erregt depressorisch auf den Tonus des gesammten Gefässsystems wirken. Stellen wir einmal an der Hand dieser Thatsachen einige Versuche an. 


\section{Sechste Versuchsreihe.}

Versuch vom 15. März 1877.

Mittelgrosser Hund. Einführung der Kanüle in den duct. Stenon. Es ist keine Speichelsecretion vorhanden. Anlegung einer Trachealfistel, um bei Durchschneidung der vagi der Dispnoe vorzubeugen. Um zu prüfen, ob die Kanüle in den Speichelgang gut eingebunden war, wird die Mundhöhle mit ein wenig verdünnter Essigsänre gereizt. Dabei tritt starke Secretion ein. Nun wird der vagus-symp. präparirt, dann durchschnitten und das centrale Ende des gemeinschaftlichen vagus-sympathicus-Stumpfes schwach elektrisch gereizt, ohne dass hierdurch eine Speichelabsonderung eingeleitet wurde. Nun wurde ein Quecksilber-Manometer in die carotis der anderen Halsseite eingebunden. Die Verbindung des Manometers, mit der in die Arterie eingebundenen Kanüle, wurde hergestellt durch eine Leitung von Bleiröhren, die mit einer starken Lösung von kohlensaurem Natron angefüllt wurden. Nach Oeffnung der Hähne steigt das Quecksilber von 0 bis auf $75 \mathrm{~mm}$. Jetzt wird der gemeinschaftliche vagus-sympathicus-Stamm mit schwachem Strom öfters gereizt und die hierbei auftretende Veränderung des Blutdruckes bietet folgendes Bild. Im Anfange der Reizung steigt das Quecksilber gewöhnlich um $5 \mathrm{~mm}$, dann sinkt es von $80-60 \mathrm{~mm}$ herab. Dieses Sinken hält einige Zeit an, dann steigt das Quecksilber wieder ganz allmählig in die Höhe. In einigen Fällen trat das Sinken ohne vorhergegangene Drucksteigerung ein und nachdem es einige Zeit angehalten hatte, stieg der Druck wieder wie vorher. Bei der Anwendung von so schwachen Strömen, die noch ein Sinken des Blutdruckes bei Reizung des centralen vagus-symp. hervorbrachten, konnte ich niemals das Eintreten von Speichelsecretion beobachten. Als ich die Stromstärke bedeutend steigerte, bot die Veränderung des Blutdruckes bei Reizung des Nervenstammes ein anderes Bild. Es trat dann weder das anfängliche schwache Ansteigen des Blutdruckes noch das Sinken ein, sondern die Quecksilbersäule stieg sofort bis auf $100 \mathrm{~mm}$. Mit dieser bedeutenden Drucksteigerung trat auch in jedem Falle eine sehr energische Speichelsecretion auf. Die Reizung des vagus war dabei schon so stark geworden, dass mit der Absonderung gleichzeitig Schlundbewegungen auftraten. Diese Bewegungen standen jedoch in keinem Zusammenhange mit der Speichelsecretion. Leider hatte ich es bei diesem Versuche versäumt auch den vagus-symp. der anderen Seite zu durchschneiden, denn die Reizung des centralen vagus-Stumpfes ruft bei intaktem vagus der anderen Seite eine Modification der Herzbewegung hervor, die den Blutdruck verändern kann. Weil ich fürchtete, dass die Veränderung des Herzschlages doch einen wesentichen Finfluss bei diesem Versuche gehabt haben konnte, so stellte ich an einem Hunde einen anderen Blutdruckversuch an mit der Durchschneidung beider vagi. Dieser Versuch bietet jedoch dasselbe Bild, wie der oben angeführte. Ich will das Versuchsprotokoll hier kurz noch mittheilen: 
Versuch vom 20. März 1877.

Durchschneidung des vagus-symp. auf beiden Seiten. Einführung des Manometers in die linke carotis. Stand der Quecksilbersäule auf $125 \mathrm{~mm}$.

Schwache Reizung des centralen vagus-symp. auf der rechten Seite. Sinken des Blutdruckes von $125-100 \mathrm{~mm}$.

Nochmalige Reizung. Sinken von $125-115 \mathrm{~mm}$.

Nochmalige Reizung. Sinken von $125-115 \mathrm{~mm}$.

Reizung mit etwas stärkerem Strom, wobei beide Spiralen des Schlittenapparates aneinander geschoben sind. Sinken von $125-110 \mathrm{~mm}$.

Reizung des centralen vagus-symp. der linken Seite. Sinken von 115 bis $100 \mathrm{~mm}$.

Nochmalige Reizung. Sinken von $125-85 \mathrm{~mm}$.

Jetzt wurden die Spiralen etwas übereinander geschoben, bis auf einen Rollenabstand von $6,5 \mathrm{~mm}$. Bei diesem starken Strome trat erst nach längerer Einwirkung der Reizung ein Sinken des Blutdruckes um $10 \mathrm{~mm}$ ein. Bei einer zweiten Reizung aus derselben Stromstärke sank auch erst wieder nach längerer Zeit der Blutdruck von 125-120 mm.

Jetzt wurde wieder mit einem schwachen Strome gereizt bei Rollenabstand von $13,5 \mathrm{~cm}$. Es trat sofort ein Sinken des Blutdruckes von 120 bis $112 \mathrm{~mm}$ ein. Nun wurden die Spiralen des Schlittenapparates ganz übereinander geschoben und so mit dem stärksten Strome gereizt. Es musste inzwischen das Blutgerinnsel aus der Kanüle entfernt werden und das Quecksilber stand jetzt auf $85 \mathrm{~mm}$.

Reizung des centralen vagus-symp., dabei werden die Spiralen des Schlittenapparates ganz über einander geschoben. Ansteigen des Blutdruckes von 85 auf $130 \mathrm{~cm}$.

Nochmalige Reizung. Ansteigen von 85 auf $120 \mathrm{~mm}$.

Nochmalige Reizung. Ansteigen von 85 auf $125 \mathrm{~mm}$.

Reizung des vagus-symp. der rechten Seite bei Rollenabstand $14 \mathrm{~cm}$. Sinken des Blutdruckes ron $90-81 \mathrm{~mm}$.

Reizung des centralen vagus-symp. der rechten Seite. Spiralen über einander. Ansteigen des Blutdruckes von 95-140 mm.

Reizung des centralen vagus-symp. der rechten Seite bei Rollenabstand $13,3 \mathrm{~cm}$. Sinken des Blutdruckes von 95 auf $89 \mathrm{~mm}$.

Reizung des centralen vagus-symp. Spiralen übereinander. Ansteigen des Druckes von $95-130 \mathrm{~mm}$.

Reizung des centralen vagus-symp. der rechten Seite bei Rollenabstand $13,7 \mathrm{~cm}$. Steigen des Blutdruckes von $90-100 \mathrm{~mm}$.

Reizung des vagus-symp. bei Rollenabstand $11,5 \mathrm{~cm}$. Sinken des Blutdruckes von $95-85 \mathrm{~mm}$.

Reizung des vagus-symp. bei Rollenabstand $10 \mathrm{~cm}$. Sinken von 90 bis $38 \mathrm{~mm}$ 
Reizung des vagus-symp. Spiralen über einander. Steigen von 90 bis $110 \mathrm{~mm}$.

Versuch vom 16. März 1877.

Kaninchen. Einführung der Kanüle in den Speichelgang. Präparation des vagus und symp. Trachealfistel. Blutdruck in der carotis $84 \mathrm{~mm}$.

Unterbindung des symp. Blutdruck $75 \mathrm{~mm}$.

Durchschneidung des symp. unterhalb der Ligatur. Blutdruck $83 \mathrm{~mm}$.

Durchschneidung des vagus. Blutdruck $90 \mathrm{~mm}$.

Reizung des vagus und zwar des centralen Endes. Sinken des Butdruckes auf $85 \mathrm{~mm}$.

Reizung des peripheren symp. Steigen des Blutdruckes von 95-105 mm mit gleichzeitigem Eintreten der Speichelsecretion.

Führte ich bei diesem Versuche die Reizung des symp. mit so schwachem Strome aus, dass kein merkliches Ansteigen des Blutdruckes zu erkennen war, so trat auch keine Absonderung ein.

Aus diesem letzten Versuchsprotokoll lassen sich folgende Resultate ziehen. Reize ich beim Kaninchen den peripheren Theil des Hals-symp., so tritt mit der Verengerung der Arterien Erhöhung des Blutdruckes und gleichzeitig Speichelsecretion ein. Reize ich den centralen vagus-Stumpf, so folgt Depression des Gefässtonus. Durch vorher angeführte Versuche habe ich gezeigt, dass die Reizung des vagus die durch symp.-Erregung hervorgebrachte Secretion verlangsame. Leitet der symp. die Secretion allein durch seine vasomotorische Funktion ein, so hemmt auch der vagus die Absonderung allein durch seine depressorisehe Wirkung anf die Gerässe. So lässt sich dieser Hemmungsmechanismus an der Hand der Experimente leicht genug erklären.

Betrachten wir nun einmal die Schwankungen des Blutdruckes bei vagus-symp-Reizung beim Hunde. Wurden schwache Ströme angewendet, so trat anfangs ein ganz geringes Ansteigen des Blutdruckes ein. Es folgte schnelles Sinken desselben. Nachdem dieses einige Zeit angehalten, steigt der Druck wieder allmählig Sehr oft bleibt das anfängliche schwache Steigen des Blutdruckes aus und es tritt sofort starkes Sinken ein. Nachdem es einige Zeit angehalten, steigt der Druck auch hier wieder allmählig. Werden dagegen starke Ströme angewendet, so tritt stets ohne vorheriges Sinken eine rapide Steigerung des Druckes ein gefolgt von Speichelsecretion. Diese Erscheinung lässt sich nur so erklärẹn. Bei schwachen Strömen tritt anfangs durch Reizung des 
symp. Drucksteigerung ein. Durch gleichzeitige Reizung des vagus wird die Drucksteigerung aufgehoben, so dass sie in einigen Fällen nur anfangs sichtbar, in anderen dagegen sofort bis zum deutlichen Sinken des Blutdruckes herabgesetzt wird. Durch längere Einwirkung des Reizes wird die depressorische Funktion des vagus aufgehoben and nun wird die durch symp.-Reizung hervorgebrachte Druckerhöhung wieder sichtbar. Werden sehr starke Ströme angewandt, so tritt eine augenblickliche Lähmung der depressorischen Fasern des vagus ein und die nun sofort sich einstellende bedeutende Drucksteigerung ist das Resultat der alleinigen Reizung des symp. Darans folgt, dass der vagus nur so lange die Secretion hemmt, als er den Gefässtonus deprimirt. Wie die Erscheinung zu erklären ist, dass der vagus anch die Speichelsecretion während der Verdaung hemmt, werden wir in einem folgenden Kapitel erörtern.

Wenn die Reizung des symp. beim Hunde in den bei weitem meisten Fällen keine Absonderung hervorruft, so erklärt sich dieses aus der gleichzeitigen Mitreizung des vagus. Die Anwendung starker Ströme, die die depressorische Funktion des Nerven aufhebt oder die ebenso wirkende lange anhaltende Reizung wird meistens von Erfolg sein. Da der vagus sensibel ist, so ist die Beobachtung an sehr empfindlichen Hunden äusserst schwer. Die Thiere machen bei der Reizung so heftige Bewegungen oder es tritt Erbrechen ein begleitet von Speichelsecretion, dass man bei ihnen oft ganz von dem Experimente abstehen muss. Unter einer grösseren Anzahl von Versuchsobjecten wird man aber immer einige finden, die sich bei der Reizung sehr ruhig verhalten. Es lässt sich dann auch noch öfters folgende Beobachtung machen.

Reizt man den centralen vagus-sympathicus-Stumpf mit mittelstarken Strömen einige Zeit hindurch, so tritt keine Speichelsecretion ein. Bei Fortnahme der Elektroden erfolgt jedoch sofort sehr energische Absonderung. Woher diese Erscheinung? Wir sahen aus den Blutdruckversuchen, dass die Reizung öfters früher eine Wirkung auf den symp. wie auf den vagus ausuibt und ebenso, dass bereits Lähmung der depressorischen Funktion des vagus eintritt, während die pressorische des symp. noch in voller Thätigkeit ist. Es liegt also die elektrische Erregbarkeit der depressorischen Fasern des vagus in engeren Grenzen wie die der pressorischen 
des symp. Reize ich beide Nerven gleichzeitig, so wird in dem Stadium des Sinkens, bevor also die depressorischen Fasern des vagus gelähmot sind, bei Aufhören der Reizung die Nachwirkung derselben beim symp. eine längere Zeit andauernd sein wie beim vagus, es werden sich also die Gefässe in den Centren der Secretionsnerven wieder kontrahiren und so die Absonderung hervorrufen.

Sehen wir nun schliesslich noch, wieweit meine Hypothese uiber die Stellung des symp. und vagus zur parotis bereits bekannte Experimente auf diesem Gebiete erklären kann. Zuerst will ieh die eigenthümliche Wirkung des Nicotins auf die Speichelsecretion, die v. Wittich zum Gegenstande eingehender Studien machte, heranziehen. Herr Professor v. Wittich hatte die Guite mir seine Versuchsprotokolle zur Einsicht zu überlassen. Die Wirkung des Nicotins wird darin wie folgt geschildert: „Einträufeln eines Tropfens Nicotin in die Lidspalte ruft bei Kaninchen fast momentan sehr heftige allgemeine Intoxikationserscheinungen hervor: vorübergehende eyanotische Färbung der rothen Theile des Mundes des Auges. Convulsive Bewegungen aller Muskeln, Verengerung der Pupille bis zur Stecknadelkopfgrösse, Ueberfüllung der Ohrgefăsse durch Lähmung des symp. Gleichzeitig tritt bei Beginn der Intoxikation excessive Ausscheidung des Parotissecretes ein, die jedoch lange nicht so nachbaltig wie nach Calabarvergiftung bald sistirt. Reizung des symp. ruft jetzt weder eine Erweiterung der ad maximum kontrahirten Pupille noch Verengernng der Ohrgefässe noch endlich Secretion der Parotis hervor, wohl aber erfolgt letztere augenblicklich, sobald man die Elektroden des Induktionsapparates direkt auf die Drüse selbst setzt."

Zieht man die Wirkung des Nicotin auf die vasomotorischen Nerven zu Rathe, die sich anfangs in einer Reizung, dann in einer totalen Lähmung derselben äussert, so findet man leicht die Erklärung dafiư, dass das Nicotin nur beim Beginn der Vergiftung die Absonderung hervorruft. Ist vollständige Lähmung des symp. erst eingetreten, so kann auch die Reizung des Nerven keine Gefässkontraktion und folglich auch keine Speichelsecretion mear hervorrufen. Gruenhagen spricht die Ansicht aus, dass Nicotin durch centrale Trigeminusreizung die Secretion einleite. Diese Anschauung scheint mir jedoch ebenso unhaltbar, wie die Annahme 
desselben Autors, dass bei Reizung des symp. das Secret der Drüse nur durch Verengerung der Blutkapillarmaschen ausgepresst wird. Letztere hat ihre Widerlegung bereits durch den $\nabla$. Wittichschen Versuch gefunden, nach dem die Reizung des symp. nach Ausreissung des facialis erfolglos bleibt.

Dass die Reizung sensibler Nerven, z. B. des ischiadicus, der Magenschleimhaut, Speichelsecretion hervorruft, erklärt sich dadurch, dass durch sie eine allgemeine Gefässverengerung bewirkt wird. Wir haben hier denselben Vorgang, wie bei direkter sympathicusReizung.

\section{Die Secretion der parotis während der Verdaung.}

Bei Kaninchen und Hunden bemerkt man ein bis anderthalb Stunden nach der Nahrungsaufnahme eine bedeutende Speichelsecretion, die sich bei Hunden bequem nach Tropfen bestimmen lässt. Bei Kaninchen ist sie weniger stark. Lässt man die Thiere aufgespannt liegen und vermeidet jede Manipulation, die sie in heftige Aufregung versetzt, so kann man diese Absonderung unter Umständen einige Stunden lang beobachten. Sie hat jedoch durchaus keine Aehnlichkeit mit der continuirlichen Absonderung der Drüse beim Schafe, auf die ich noch zu sprechen kommen werde. Bei diesem Thiere bleibt in gleichen Zeiträumen die abgesonderte Speichelmenge ziemlich gleichmässig gross, während sie beim Hunde und Kaninchen allmählig abnimmt, endlich so klein wird, dass man sie nur noch im feinen Glaskapillarrohr beobachten kann. Zuletzt tritt gänzlicher Stillstand ein. Ist der Versuch rein, macht das Thier keine starken Bewegungen besonders der Kaumuskeln, so wird man niemals ein stossweises Ansteigen der Speichelsäule, sondern nur ein allmähliges Fortschreiten derselben beobachten.

Es fragt sich nun, wodurch wird beim Hunde und Kaninchen während der Verdauung dieșe Șecretion eingeleitet. Das natïr- 
lichste scheint zu sein, dass die im Magen angehäuften Speisemassen die Wände desselben reizen und so durch Vermittelung des symp. die Absonderung hervorrufen. Durchschneidet man jedoch den Hals-symp., so geht die Speichelsecretion nach momentaner Vermehrung ruhig ihren Gang weiter, höchstens wird sie um ein klein wenig verlangsamt, wodurch werden wir später sehen. Man könnte vielleicht denken, dass nach Durchschneidung des Halsstranges des symp. die Reizung vom Magen aus dureh den ihm zugelegenen Theil des Nerven durch seine Verbindungsäste mit dem Halsmarke in dieses und ron da wieder weiter fortgeleitet werden könnte. Dieses ist jedoch nicht möglich. Wäre es der Fall, so müsste die Reizung des centralen Theiles des Hals-symp. auch eine Absonderung hervorbringen, ich habe dieses jedoch, so oft ich es auch versucht habe, nicht beobachten können. Wir müssen also annehmen, dass während der Verdauung Bedingungen in dem Organismus geschaffen werden, die im Stande sind den secretorischen Nervenapparat zu seiner Funktion anzuregen. Aufschluss giebt uns folgender Versuch.

Versuch vom 22. November 1876.

Hund. Um $1^{1} j_{4}$ Uhr hat er stark gefressen. Tracheotomie. Einführung der Kanüle in den Speichelgang. Um 3\% Uhr wird die Menge des secernirten Speichels bestimmt.

$$
\begin{aligned}
& \text { In } 1 \text { Min. . . . . }-7 \text { Tropfen. } \\
& " 1 " . . .+8 \% \\
& " 1 " . .5 \% \\
& n 1 n . . .5-8 n
\end{aligned}
$$

Einleitung künstlicher Respiration. Ohne Unterbrechung wird die Secretionsgrösse während derselben weiter bestimmt.

In 1 Min. . . . . - 5 Tropfen.

$$
\begin{aligned}
& " 1 " . .50 \\
& " 1 \% \text {. . . - } 3 \text { - } 3 \\
& " 1 " \cdot . \cdot .-1 \% \\
& " 1 " 5 \text { sec. }-1 \text { - } 1
\end{aligned}
$$

Sistirung der künstlichen Athmung.

$$
\begin{aligned}
& \text { In } 1 \text { Min. . . . . } 8 \text { Tropfen. } \\
& " 1 \% \text {. . . . }-11 \% \\
& " 1 \% \cdot \cdot_{\varepsilon} \cdot \cdot-8 \% \\
& " 1 \% \text {. . . . }-7 \text {, } 7 \\
& \text { " } 1 . \cdot . \cdot-11 \text {. }
\end{aligned}
$$


Einleitung der künstlichen Athmung.

Sistirung der künstlichen Athmung.

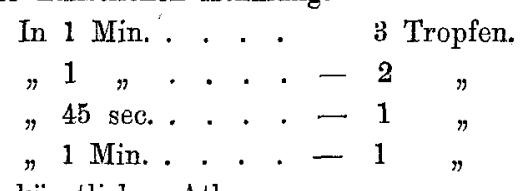

In 1 Min. . . . . - 14 Tropfen.

Versuch vom 1. Dezember 1876.

Hund. Tracheotomie.

Ohne künstliche Respiration.

Mit künstlicher Respiration.

In 1 Min. . . . - 7 Tropfen.

"1 $" . .5 \%$

In 1 Min. . . . . - 4 Tropfen.

Ohne künstliche Respiration.

$" 1$ " . . . -2 "

In 1 Min. . . . . - 5 Tropfen.

Nach einiger Zeit, als die Verdauungsspeichelsecretion schwächer geworden war, bekam ich folgendes Resultat.

Ohne künstliche Respiration.

$$
\begin{aligned}
& \text { In } 1 \text { Min. . . . . - } 4 \text { Tropfen. } \\
& \text { " } 1 \text { " } 5 \text { Sec. . - } 4 \text { " }
\end{aligned}
$$

Mit künstlicher Respiration.

In 4 Min. . . . . -1 Tropfen.

Ohne künstliche Respiration.

In 1 Min. . . . . -2 Tropfen.

Vergleichen wir mit diesen Versuchsresultaten eine Angabe von E. Becher, Zeitschrift für rationelle Medicin Band 6, Heft 3, der den Kohlensäuregehalt des Blutes zu verschiedenen Tageszeiten bestimmte, so ist sofort klar, dass auch die Speichelsecretion bei der Verdauung eingeleitet wird durch den grösseren Kohlensäuregehalt des Blutes. Daher bewirkt künstliche Respiration bei diesem Vorgange eine Verlangsamung der Absonderung; die mit jeder Bewegung des Blasebalges immer schwächer wird, ja sogar ganz sistirt werden kann.

Nach Becher enthielten 100 volum. der Exspirationsluft um 9 Uhr Morgens nüchtern

Um 11 Uhr niichtern

$$
6,692 \% \mathrm{CO}_{2} .
$$

$6,680 \% \mathrm{CO}_{2}$. 
Um 2 Uhr Mittags 1/1/2 Stunde nach dem Essen

Um 4 Uhr Nachmittags

$$
7,123 \% \mathrm{CO}_{2} \text {. }
$$

Um 6 Uhr Abends

$$
7,074 \% \quad \mathrm{CO}_{2} \text {. }
$$

$$
7,047 \% \mathrm{CO}_{2} \text {. }
$$

Es wird jetzt auch keiner weiteren Erklärung bediurfen, dass die Durchschneidung des symp. und die Reizung des vagus die Speichelsecretion während der Verdauung zu verlangsamen im Stande ist. Durch beide Mittel erleichtern wir die Zufuhr von arteriellem Blut in das secretorische Nervencentrum. Es ist klar, dass auch jeder andere Nerv, der gereizt depressorisch anf den Tonus der Gehirngefässe wirkt, eine Verlangsamung der Speichelabsonderung hervorzubringen im Stande sein wird.

Ich will noch bemerken, dass man durchaus nicht erwarten darf, bei jedem Hunde, der sich tüchtig vollgefressen hat, die Speichelabsonderung während der Verdauung and den Einfluss der vagus-Reizung auf sie klar beobachten zu können. Sehr oft ruft die Operation der Isolirung des vagus schon Sistirung der Absonderung hervor, die bald darauf wieder durch psychische Erregung und heftige Bewegungen des Thieres eingeleitet werden kann. In diesem Falle ist selbstverständlich die Einleitung der künstlichen Respiration nicht nur ohne verlangsamenden Einfluss, sondern es wird auch der neue Eingriff bei empfindlichen Objekten den Erregungszustand erhöhen und so die Absonderung vermehren. Ich glaube nicht, dass es nöthig sein wird aus meinen Versuchen hierfuir noch Beispiele mit Zahlen anzuführen. Die Thiere arbeiten dann krampfhaft der künstlichen Athmung entgegen. Auch daran lässt sich diese Art der Absonderung erkennen, dass sie an Grösse bedeutend schwankt, bald vollständig stillsteht, um wieder in der nächsten Zeit mit erneuter Energie aufzutreten. 


\section{Die Secretion der parotis des Schafes.}

In der Voraussetzung, dass vielleicht auch beim Schafe der Kohlensäuregehalt des Blutes die Speichelabsonderung beeinflussen könnte, stellte ich an vier verschiedenen Objekten Versuche mit künstlicher Respiration an. In keinem Falle konnte ich auch nur die geringste Wirkung derselben auf den Vorgang in der Drüse konstatiren. Es ist dieses nur entsprechend der Thatsache, dass beim Schafe die Secretion weder vom symp. noch von einem der Gehirnnerven beeinflusst wird. Wir müssen jetzt wohl annehmen, dass bei diesem Thiere die Speichelsecretion nur durch eine specifische Energie der Drüsenzellen zu Stande kommt.

Stellen wir zum Schluss noch einmal kurz die wesentlichen Resultate meiner Untersuchungen zusammen:

Der Kohlensäuregehalt des Blutes spielt wie auf dem Gebiete anderer Nerven so auch bei den Secretionsnerven als Reizmittel eine grosse Rolle. Auf diese Weise wird die Speichelsecretion während der Verdaung und auch bei sympathicus-Reizung eingeleitet. Der symp. führt weder secretionsbefördernde noch secretionshemmende Nervenfasem. Ein hemmender Einfluss auf die Speichelsecretion bei sympathicus-Reizung und der Verdaung. kommt dem n. vagus zu. Aber auch dieser Nerv führt keine besonderen secretionshemmenden Fasern, sondern er ist nur wirksam durch seine vasomotorische Funktion. Beim Schafe hat der Kohlensäuregehalt des Blutes keinen Einfluss auf die Absonderung der parotis. Für das ungleich wichtigste Resultat halte ich es, dass die Speicheldrüse uns Gelegenheit gegeben hat, einen Einblick in einen Vorgang zu erhalten, den man ohne diese Kenntniss mit dem Namen eines Reflexvorganges belegen musste. Ebenso haben wir die Natur eines Hemmungsmechanismus im Nervengebiete erkennen können. Durch Heranziehung des RosenthalUspensky'schen Versuches sahen wir, dass es nicht nöthig ist anzunehmen, dass die bei sympathicus-Reizung auftretende Verlangsamung des Herzschlages bedingt sei durch eine Reflexwirkung: dieses Nerven auf den vagus. Es wird wohl der symp. in demselben Verhältniss zum vagus wie zum facialis stehen! Beide Nerven werden durch die bei sympathicus-Reizung sich einstellende 
214 Adolph Jaenicke: Untersuch. üb. d. Secretion d. glandula parotis.

Gefässkontraktion mit folgender Stagnation des venösen Blutes zu ihrer Funktion angeregt. Es ist wohl nicht zu gewagt anzunehmen, dass noch manche Erscheinungen bei Reizung des sympathicus und sensibler Nerven, die wir jetzt zu den Hemmung's- und Reflexvorgängen rechnen, bedingt sind durch die dabei auftretende Gefässdilatation und Kontraktion. -

Augenblicklich mit der glandula submaxillaris beschäftigt, hoffe ich auch die Resultate hiervon mittheilen zu können. -

Zum Schluss den Herren Prof. v. Wittich and Dr. Oscar Langendorff den besten Dank für die von ihnen mir gütigst gewährte Unterstïtzung. 